\title{
Improving ecological outcomes by refining decision support tools: A case study using the Murray Flow Assessment Tool and the Sustainable Rivers Audit
}

\author{
$\underline{\text { R.E. Lester }}^{\mathrm{a}}$, C.A. Pollino ${ }^{\mathrm{b}}$, and C.R. Cummings ${ }^{\mathrm{a}}$ \\ ${ }^{a}$ School of Life \& Environmental Sciences, Deakin University, Warrnambool, Victoria, Australia \\ ${ }^{b}$ CSIRO Land and Water, Canberra, ACT, Australia
}

Email: rebecca.lester@deakin.edu.au

\begin{abstract}
Robust decision support tools are critical to assist stakeholders to maximise the ecological benefit of their management strategies. Unfortunately, the development phase for decision support tools can often be considered an endpoint by stakeholders and insufficient testing and validation is a common trait for applied ecological models. The Murray Flow Assessment Tool (MFAT) divided a range of water-dependent taxa, including fish, into functional groups (e.g. flood spawners, wetland specialists) and used available literature and expert opinion to derive a range of response curves for relevant flow-related habitat conditions. Response curves were developed for variables such as flow and spawning timing and flow duration for fish, and were spatially differentiated where considered appropriate. While the development used best-available science, the level of confidence reported for many of these response curves was moderate or low, the sensitivity of the model to the method used to combine response curves could not be tested in the development timeframe and subsequent validation of model predictions has been minimal, to our knowledge. Weightings applied to the various component parts of a habitat condition score have also not been assessed.
\end{abstract}

Here, we use fish data collected as a part of the Murray-Darling Basin Authority's Sustainable Rivers Audit (SRA) to begin to understand how well MFAT habitat condition scores relate to measured biological data. We compare whether fish species within functional groups respond similarly, whether the spatial differences captured by MFAT for fish are reflected in the SRA data and make a first assessment of how the method of combining individual response curves and then the weightings applied to each affect the final prediction, and then how that compares with measured fish data.

Overall, we detected a low level of correlation between MFAT habitat condition scores and fish assemblages as measured by SRA. Individual model metrics for the various fish functional groups calculated by MFAT were not better correlated with measured fish population metrics for the corresponding functional groups calculated from SRA data. Main channel generalists were the best correlated individual group, and they were also the most abundant within the SRA data. However, little improvement in correlation was observed for the overall groups of total, native and invasive fish richness, abundance or biomass and the overall MFAT score was negatively correlated with those fish metrics in all years except for 2002. Overall measures of fish assemblage were better correlated with individual components of the MFAT score, including native richness and abundance which were moderately correlated with main channel generalist adult habitat condition, an index largely based on non-flow related parameters such as availability of woody debris. Introducing lags in fish response time did not improve the relatively-weak correlations.

Methods of combining response curves resulted in large changes in the scores derived from MFAT, and the variability of combination methods across fish functional groups and locations is having an impact on the simulated habitat condition. The implications of this are unclear. Furthermore, the default weightings applied to each component did not influence the strength of the correlations observed with SRA data, with constant equal weightings and, for at least one zone, randomly-generated weightings, resulting in similar or better correlations. Thus, we recommend the use of constant equal weightings as a simplification to the MFAT model, and that the method of combining components be revisited with a view to increasing the consistency across the zones. We also recommend additional validation of the model using data encompassing a wider range of flows than were available for this assessment.

Keywords: Habitat suitability model, Ecological response model, Preference curve, Evaluation. 


\section{INTRODUCTION}

A range of modelling approaches has been used to assess the possible ecological outcomes of flow regimes. Habitat suitability models (HSMs) are generally static representations of change, which are often spatial and probabilistic in nature. They serve three main purposes: to predict species occurrence on the basis of abiotic and biotic variables; to improve the understanding of species-habitat relationships; and to quantify habitat requirements (Ahmadi-Nedushan et al., 2006). Habitat suitability is often expressed as a suitability curve (e.g. suitability or preference curve), which specifies the assumed requirements of a species, life stage or guild (e.g. Lamouroux et al., 1998). Curves are used to depict an organism's response to a changing habitat variable (often as an index of 0 to 1). Three types of habitat suitability indices (HSIs) were distinguished by Bovee (1986, as cited by Jowett et al. (2005)): professional judgement; habitat use; and habitat preference indices. The first are derived from life-history studies in the literature or expert judgement. The second use data on the frequency of occurrence of actual conditions used by a species. The final type combines frequency analysis with habitat availability data for sampled reaches. HSIs can be created separately for different variables (e.g. low and high flows) and then combined to define a composite index.

A broad range of modelling tools has been developed to predict how physical habitat conditions (i.e. water depth, velocity, cover, substrata) change with discharge. The Instream Flow Incremental Methodology (Bovee, 1982), and its associated software the Physical Habitat Simulation System (PHABSIM; Shuler and Nehring, 1993), are widely used HSMs. Typically, these compare hydrological and hydraulic data for a grid of cells in a river stretch with the HSIs of a target species. The Murray Flow Assessment tool (MFAT) (Young et al., 2000) uses similar modelling to PHABSIM, where habitat requirements are modelled as preference curves. More recent incarnations of the approach can be found in EcoModeller (Marsh et al., 2007) and the IBIS Decision Support System (Merritt et al., 2010). These models use habitat suitability indices derived using professional judgement, using the classification of Bovee (1986).

In HSMs, the most common methods used for combining preference curves to calculate a composite HSI are the arithmetic mean, geometric mean ( $n$th root of the product of $n$ individual indices) and the most limiting factor. In EcoModeller, the most limiting factor is used (Marsh et al., 2007), whereas IBIS allows comparison of an arithmetic mean and an expert-driven weighting process (Merritt et al., 2010). MFAT uses a range of combination methods, which were derived from expert judgements made by the Regional Evaluation Groups (REGs) (Young et al., 2003). PHABSIM enables the user to select the composite algorithm, where input variables can be unweighted or they can be weighted.

MFAT was developed to assess the ecological response to flow scenarios at a range of locations along the River Murray (Young et al., 2003). Daily river flows were used as input to assess algal growth, in-channel habitat condition for native fish and associated off-channel habitat condition for wetland and floodplain vegetation and waterbirds. Seven zones (excluding the River Murray estuary) were included. Within each zone, REGs developed HSIs for each functional group for typically 2 to 3 regions. At the time of development, MFAT was considered to capture and document the best available ecological and hydrological scientific knowledge for the Murray and provide a mechanism for assessing the ecological outcome of flow scenarios, providing a repeatable and objective comparison integrating across a range of scales (SRP, 2003).

The process used to develop an index of fish habitat condition is typical of the process used for other taxa. A range of response curves were developed for each functional group for flow-related variables (e.g. inundation duration, dry period) which were then combined to give an assessment of adult habitat condition (AHC) and recruitment habitat condition (RHC) for each functional group. These were again combined into a fish habitat condition (FHC) index that was aggregated across functional groups to give a single fish HSI per sub-region. Weightings and combination methods at each step were determined using the expert opinion of the REGs.

The limitations of MFAT are similar to those for other HSMs where outcomes consider single events in isolation rather than dynamic change and are often based on limited data or poorly-documented thresholds (Miao et al., 2009; although MFAT does have extensive documentation). It relies on expert opinion, with the fallacies inherent therein (Cooke, 1991) and tends to ignore the potential response to multiple extreme events or event connectivity (Miao et al., 2009). HSMs also tend to lack representation of interactions between species, life stages and the effects of the range of habitat variables (Ayllón et al., 2011). MFAT specifically is based on hydrologic data, not hydraulic data, so does not consider depth, velocity or substrate, all of which may be more influential than flow per se (SRP, 2003) thus limiting the small-scale conclusions that can be drawn. In some instances, MFAT has been criticised for not being easy to use and for being overly complex, largely through the number of weightings and the methods of combine preference curves used. However, MFAT was considered to be a work in progress following its initial development (SRP, 2003) and, to our knowledge, no further development has occurred, and this assessment stands. Furthermore no other tools 
have been developed to supersede MFAT, so there is benefit in attempting to test the model structure.

Our aim was to test the fish habitat condition scores generated by MFAT against fish assemblage data that are now available for the MFAT-defined zones to identify whether the two were correlated, thus investigating one of the main purposes of a HIS: that it predicts species occurrence based on biotic and biotic factors. We describe which aspects of current assemblage data were in line with the results of MFAT simulations, identify where MFAT was performing well, and make recommendations for improvement where it was not. We also investigate the sensitivity of the model to combination methods and variable weightings, effectively exploring the manner in which suitable habitat is defined for the fish functional groups used within MFAT.

\section{METHODS}

\subsection{Fish assemblage data for comparison with MFAT habitat condition scores}

The fish data used in this paper were sourced from the Sustainable Rivers Audit (SRA), which is a program to assess the condition of river ecosystems in the MurrayDarling Basin (Davies et al., 2010). The SRA sample data record the identity, origin and condition of individuals, as well as the abundance, biomass and community composition (as species). We use the abundance, biomass and species richness for each

Table 1. Summary of SRA fish data showing richness (abundance) for each of the MFAT fish functional groups. Refer to text for abbreviations.

\begin{tabular}{|l|r|r|r|r|}
\hline \multirow{2}{*}{} & \multicolumn{2}{|c|}{ Mitta Mitta River (Zone A) } & \multicolumn{2}{c|}{ Murray River (Zone C) } \\
\cline { 2 - 5 } & Colemans & \multicolumn{1}{c|}{ Tallandoon } & Yarrawonga & \multicolumn{1}{c|}{ Tocumwal } \\
\hline FL spawn & $7(20)$ & $3(6)$ & $1(1)$ & $0(0)$ \\
\hline Mac perch & $0(0)$ & $1(2)$ & $0(0)$ & $0(0)$ \\
\hline Wet spec & $11(75)$ & $4(41)$ & $2(206)$ & $1(6)$ \\
\hline FW catfish & $0(0)$ & $0(0)$ & $0(0)$ & $0(0)$ \\
\hline MC gen & $23(130)$ & $3(40)$ & $2(206)$ & $2(40)$ \\
\hline MC spec & $9(77)$ & $9(46)$ & $8(126)$ & $12(206)$ \\
\hline LF spec & $2(13)$ & $2(3)$ & $0(0)$ & $0(0)$ \\
\hline Other & $13(332)$ & $5(173)$ & $8(375)$ & $14(252)$ \\
\hline Invasive & $77(1305)$ & $31(415)$ & $25(864)$ & $58(944)$ \\
\hline
\end{tabular}

sampling location within the zones defined by MFAT in our analyses. SRA fish sampling used standardised methodologies to minimise errors due to variation in catch effort. This paper uses data obtained over 20042006. Only electrofishing data were included to minimise variability due to sampling method.

Species were grouped according to MFAT functional groups (i.e. flood spawners [FL spawn], Macquarie perch [Mac perch], wetland specialists [Wet spec], freshwater catfish [FW catfish], main channel generalists [MC gen], main channel specialists [MC spec] and low-flow specialists [LF spec]), with total, native, invasive and other groups also created (i.e. invasive and other are not in MFAT). Abundance, biomass and species richness were calculated for each ('fish metrics'; Table 1).

\subsection{Modelling a baseline hydrological scenario}

The hydrological scenario used as input to these analyses was a current river operations baseline (CSIRO 2008). The outputs represent a scenario from MSM -Bigmod, which provides daily flows in the Murray River over $114 \mathrm{y}$. Daily hydrologic outputs were used as inputs to the MFAT ecologic models in two zones. Basic flow metrics (Table 2) were used to describe the scenario at the model flow input locations, for both the entire model period and the period over which SRA data were collected. Colemans and Tallandoon are located in the upper and lower Mitta Mitta River respectively, which makes up the headwaters of the Murray River (Zone A). Yarrawonga and Tocumwal are found in the mid-lowland reaches of the Murray (Zone C).

\subsection{Evaluating correlations between MFAT scores and fish metrics}

Correlations between the overall MFAT score and fish metrics were evaluated for the two MFAT zones modelled. Pearson's $R$ correlation coefficients were generated using SYSTAT v. 12. Correlations were also generated between the same fish metrics and the components of the MFAT habitat condition score: FHC, AHC and larval habitat condition (LHC), which was the component of RHC which varied most.

We tested whether it was most appropriate to use the MFAT habitat condition score for the current year as a measure of fish metrics in the same year by correlating the same suite of scores from the ten years prior to fish data collection, to identify any consistent lags in response time. We also investigated whether previous calendar years (i.e. regardless of the year in which fish were sampled) were more likely to be strongly correlated, again for the ten years prior to the final survey in 2006, to determine whether there were any threshold (or other year-specific) responses not captured by a uniform lag in time until fish response. 


\subsection{Evaluating the influence of weightings on the MFAT score}

In order to test the influence of the weightings applied to each components on the overall MFAT score obtained, we compared three weighting types. Default combinations, reflecting the expert inputs of the REGS, constant weightings, where all factors were treated equally, and random numbers (generated in Microsoft Excel) were all used. Weightings were adjusted for AHC and RHC, which were used to calculate FHC. Weightings were also altered for the RHC inputs; spawning habitat condition (SHC) and LHC. These scores were hydrologically-defined, where inputs included flood magnitude, flow timing, rate of flow rise and fall, duration of flow, flushing flows, flow percentiles and no-flow conditions. The AHC component is dependent on physical habitat variables (e.g. woody debris, water temperature, channel condition), fish passage and maintenance flows. These variables were fixed over the duration of the modelling period. The overall MFAT score represents the aggregate FHC for all functional groups, equally weighted.

\subsection{Evaluating the influence of the method of combining component scores}

In order to test the influence of the method of combining the components of each score on the overall MFAT score, we compared six common methods of combining response curves with that used for LHC for main channel specialists in Zone $\mathrm{C}$ for the Yarrawonga to Tocumwal stretch of the Murray River. By default, LHC for main channel generalists is a function of flow duration (FD), inundation area (IA), inundation duration (ID), dry period (DP) and flow percentile $\left(\mathrm{FP}_{\mathrm{L}}\right)$. These were combined according to the following formulae:

LHC $=\sqrt{F F_{E} \times F D}$ where the median flow is less than the natural median, and $\mathrm{LHC}=\sqrt[3]{F A \times W D \times D F}$ where the median flow is greater than the natural median.

We calculated LHC using a maximum (max), minimum (min), average (ave), geometric mean of $\mathrm{FP}_{\mathrm{L}}$ and FD (sqrt flows), constant of 1 (constant used in MFAT for wetland specialist fish), and multiplying $\mathrm{FP}_{\mathrm{L}}$ and FD (multi flows). We report the percentage change in LHC, RHC and FHC compared with the defaults.

\section{RESULTS}

\subsection{Evaluating correlations between MFAT scores and SRA fish data}

Overall MFAT scores were poorly correlated with SRA overall fish metrics ( $n=101$ for all correlations). The best correlation from among that set was with total abundance ( $R=0.15$ with a 3 -y lag). Without a lag, the MFAT score tended to be moderately negatively correlated with overall fish assemblage metrics (e.g. $R=$ -0.57 for total abundance).

The overall MFAT habitat condition score was best correlated with the richness $(R=0.61$ with a 9-y lag), abundance $(R=0.57$ with a $9-y$ lag) and biomass $(R=0.42$ with a 9 -y lag) of the main channel generalists. Shorter lags were also relatively well correlated, with lags of 2, 5 and 7 years showing slightly weaker correlations than the 9 year lag. MFAT scores were also weakly correlated with wetland specialist richness $(R=0.32$ with a 10-y lag) richness and biomass $(\mathrm{R}=0.39$ with a $10-\mathrm{y}$ lag) and main channel specialist

Table 2. Basic flow metrics (ML/day) for the baseline scenario for the two regions analysed herein. Yarrawonga is downstream of the weir at that location. All metrics are for the 114-year run unless specified.

\begin{tabular}{|l|r|r|r|r|}
\hline \multirow{2}{*}{} & \multicolumn{2}{|c|}{ Mitta Mitta River (Zone A) } & \multicolumn{2}{c|}{ Murray River (Zone C) } \\
\cline { 2 - 5 } & Colemans & Tallandoon & Yarrawonga & Tocumwal \\
\hline Minimum & 0 & 71 & 1759 & 1786 \\
\hline Maximum & 36023 & 69279 & 198442 & 205326 \\
\hline Mean & 2488 & 3397 & 13544 & 13582 \\
\hline Median & 898 & 1825 & 9880 & 9992 \\
\hline 2004-06 Mean & 3046 & 3576 & 9943 & 9906 \\
\hline 2004-06 Median & 400 & 1572 & 9991 & 9919 \\
\hline
\end{tabular}
richness $(R=0.31$ with a 10 -y lag) and abundance $(R=0.30$ with a 10 -y lag). With no lag, MFAT overall was best correlated with main channel generalist richness $(\mathrm{R}=0.44)$ with main channel generalist abundance next best correlated. No analyses were possible for freshwater catfish as no individuals were collected during sampling.

Of the various components of the MFAT score, across the 10 years prior to fish sampling, FHC showed very few correlations over 0.3 , and the FHC for a given functional group was never correlated with the abundance, richness or biomass of that group with a Pearson's $R$ over 0.3 , regardless of the lag included.

AHC varied little across years as most components were non-flow related (so only the most common 
correlation coefficient is reported). Main channel generalist richness was moderately strongly but negatively correlated with AHC for that group $(R=-0.65)$, with similar but weaker relationships for abundance and biomass. No other metrics were correlated with the corresponding AHC, and a number of the AHC scores were uniformly negatively (but often weakly) correlated with all fish metrics. However, some overall fish metrics were better correlated with individual functional-group AHC scores than with MFAT overall. Main channel generalist AHC was moderately correlated with native fish abundance and richness $(R=0.57$ and $R$ $=0.49$, respectively, both with a $1-\mathrm{y}$ lag $)$. The relationship with total richness was stronger $(R=0.79$ with a $1-y$ lag), but appeared to be driven by a strong correlation with invasive richness ( $R=0.81$ with a $1-\mathrm{y}$ lag).

RHC did vary among years, but there were no good correlations between the specific RHC for most functional groups and their metrics. Main channel generalists were again an exception, with richness moderately correlated with its RHC (e.g. $R=0.64$ with no lag). Again, overall fish metrics were better correlated with individual RHC scores than the corresponding functional group. Low-flow specialist RHC was moderately correlated with total and invasive richness $(R=0.86$ and $R=0.83$, respectively, both with 9y lags), and invasive abundance $(R=0.83$ with a $5-y$ lag, or 0.81 with a $9-\mathrm{y}$ lag). Main-channel specialist RHC showed a very similar pattern.

Correlations across calendar years were also investigated in case there were flow characteristics associated with a particular year that led to MFAT scores that were better correlated with fish metrics. MFAT overall was consistently better-correlated with fish metrics in 2002 than any other year. While all correlations in that year were relatively weak, correlations with MFAT scores from other years

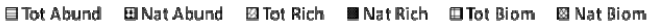

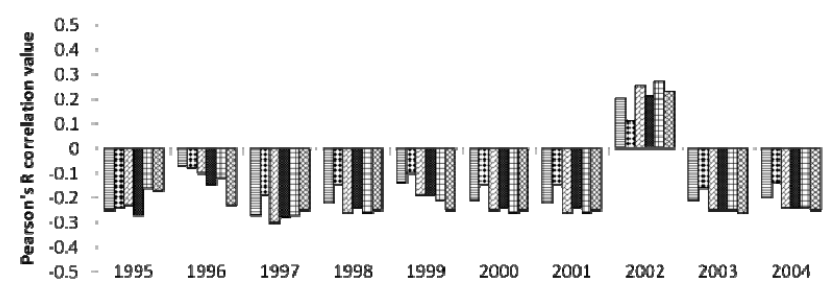

Figure 1. Correlations between overall MFAT scores and fish metrics by calendar year

were uniformly negative (Figure 1). This pattern was consistent across all functional groups, except for main channel specialists and the other fish category, which was not included in MFAT.

This pattern of one particular year being more strongly correlated with fish metrics was not observed for the components of MFAT. There was no obvious trend for FHC, AHC or RHC in one calendar year to be more strongly correlated with fish metrics than any other and most correlations were very weak.

\subsection{Evaluating the influence of weightings on the MFAT score}

The weightings used to combine the components did not influence the strength of correlations observed between MFAT score and fish assemblage metrics. For Zone A, there was less than a 0.01 change between the correlations observed for metrics derived using the defaults compared with constant equal weightings. In Zone $\mathrm{C}$, the same comparison resulted in less than a 0.03 change. When the defaults were compared to randomly-generated weightings in Zone $\mathrm{A}$, there

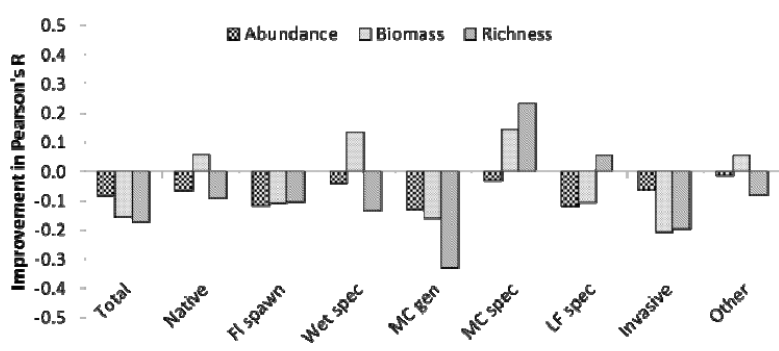

Figure 2. Change in correlations with fish metrics for randomly-generated component weightings compared with default MFAT weightings. Mac perch and FW catfish groups were omitted due to low catch rates. was a consistent improvement in the correlation between the randomly-generated weightings and fish metrics, compared to the observed correlations. For the most part, this shifted the weightings from moderate to slight negative correlations observed under the original weightings (with no time lag introduced) to or a very small negative correlation or effectively no relationship. In Zone $\mathrm{C}$, the effect of random weightings was more variable (Figure 2). Here, the changes in Pearson's $R$ were larger, but were both positive and negative, depending on the group. More common groups (e.g. total and main channel generalists) tended to perform better with the default than the random weightings, and changes in Pearson's $R$ were up to 0.3 .

\subsection{Evaluating the influence of the method of combining component scores}

The method of combining preference curves had a large impact on the value of LHC obtained. Four of the six alternative combination methods applied tended to decrease LHC, while the other two resulted in increases (Figure 3). Changes of $+50 \%$ and $-100 \%$ in LHC were observed for individual years within the 114-year model run. On average, altering the combination method for LHC resulted in changes of between - 
$100 \%$ and $+23 \%$. When propagated through the model to calculate RHC and FHC, this impact was progressively lower, due to the impact of other factors in those calculations which we did not recalculate.

\section{DISCUSSION}

Overall MFAT scores were poorly correlated with the fish assemblages sampled during the Sustainable Rivers Audit in the upper and lower Mitta Mitta and Yarrawonga to Edwards River catchments. This suggests that either habitat suitability is poorly defined within the model, or that habitat is not the primary driver of the measured fish assemblages. There was little evidence that this lack of correlation was due to a lag in the response of fish assemblages, unless that lag was longer than the 10 years investigated. There was also little evidence that FHC scores were correlated with metrics describing the functional group they were designed to represent. The exception was for main channel generalists, which tended to be the best correlated group across the range of MFAT score components investigated. This may have been because that group was the most abundant during SRA sampling, while some of the other functional groups were less abundant. Biomass was the least well correlated of the three measures of fish assemblage. Richness and abundance were similarly correlated across all the functional groups investigated. There was also a trend for the overall MFAT score in 2002 to be the best correlated for the majority of overall and functional group metrics.

Correlations between MFAT scores and SRA data provide one measure of the translation of MFAT habitat condition into fish assemblage metrics. The correlations presented here need to be interpreted cautiously as only four locations were used (i.e. two per zone), so there was limited variability in the MFAT scores for each year. The years in which fish data were collected (2004-2006) were low-flow years, so the model's ability to perform under a range of flows was not assessed. Where weak or moderate correlations were apparent, longer lags (i.e. 9-10 y) for overall MFAT scores were generally better correlated with fish metrics than shorter lags, possibly due to higher flows in those years. The unevenness in the catch associated with each group may also have contributed to relatively poor correlations for individual functional groups, but this should not have affected overall fish metrics. Investigating additional zones would resolve this issue as it would add more individuals to most groups.

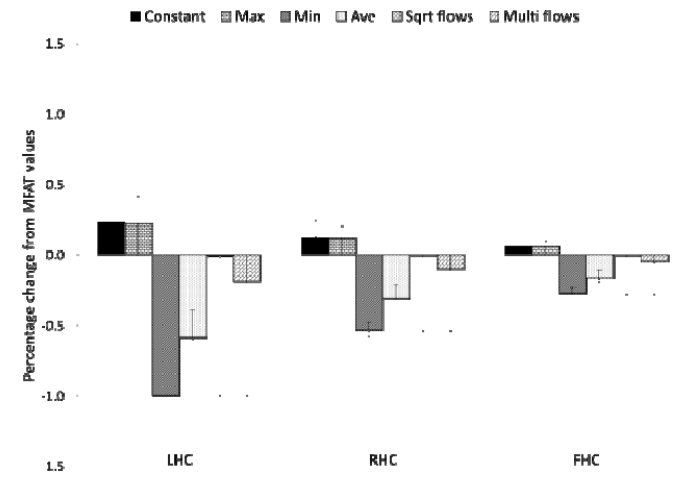

Figure 3. Percentage change in LHC, RHC and $\mathrm{FHC}$ from the default for $\mathrm{MC}$ spec in Zone C. Bars show average change over a 114-year model run, with error bars showing the observed range. See text for abbreviations.

The trend for the overall MFAT score from 2002 to be much better correlated than any other year, although the correlations were still weak. This trend was not replicated in any of the MFAT components, so it is difficult to determine what is driving the pattern; however, the modelled hydrology shows that 2002 was the second consecutive year without a peak flow. Given the low flow conditions in 2001-2002, the River Murray at the sites examined may be acting as refugia. Coincidentally, MFAT was developed in 2002/03, so it may also be that the calibration process has led to a model that is less generalisable across other years than intended, possibly due to the indirect effects of water quality which are likely to be influential in structuring fish assemblages in low-flow years (Bice, 2010) but are not explicit in the model (SRP, 2003). This may explain why the structural components of MFAT (i.e. captured in AHC) tended to be better correlated with overall fish metrics. Should this pattern be apparent upon further testing of the model, it may indicate that flow is less important than habitat structure for the period examined.

The weightings used to combine individual MFAT components into an overall score appeared to add little to the correlation of model outputs with fish metrics. In fact, the randomly-generated weightings performed best out of the three in Zone A. In Zone C, the actual weightings performed better than random weightings, particularly for the more common functional groups, but there was no difference between the default and using constant equal weightings. While the default weightings were assigned based on expert opinion regarding the most important habitat elements for the various functional groups, this preliminary analysis suggests that constant weightings would perform equally well, and are a potential simplification.

The method of combining response curves had a substantial impact on the overall score obtained. Here, we only altered one group of fish, in one section of the river, and observed changes of up to $28 \%$ in FHC. Given that some reaches only include two or three fish groups, this has the potential to substantially affect the overall MFAT score, particularly if changes interact among groups. Previous modelling found differences of between $15 \%$ and $36 \%$ as a result of an additional $1500 \mathrm{GL} \mathrm{y}^{-1}$, (SRP, 2003) so this difference is likely to be 
ecologically meaningful. Thus, careful consideration must be given to the method chosen. We would recommend combinations be as simple as possible, given the potential for unintended interactions with more complex expressions. Other similar HSMs use arithmetic or geometric means, multiplicative combinations or limiting factors (i.e. either a maximum or minimum). There are assumptions associated with each (e.g. that habitat condition of one variable such as velocity can compensate to some degree for poor condition in another such as depth for an arithmetic mean or geometric mean (Korman et al., 1994), or no compensation for a limiting factor). The combinations used in MFAT vary substantially between fish functional groups and between zones, and many are complex equations. This inconsistency is having an impact on the MFAT scores produced for each zone and the implications of this are not clear.

\section{CONCLUSIONS AND RECOMMENDATIONS}

MFAT was poorly correlated with fish metrics derived from SRA fish surveys in 2004 to 2006 for the upper and lower Mitta Mitta River and Murray catchments between Yarrawonga and the Edward River. Structural components were better correlated than flow-related components and main channel generalists were the group best explained. Default weightings on components did not improve the correlation of MFAT with fish metrics over constant equal weightings or over randomly-derived weightings for one zone. The method of combining metrics is having a large impact on MFAT scores and inconsistencies across functional groups and zones are likely to be affecting the habitat condition assessment, with the implications of this unclear.

We recommend that constant equal weightings are used instead of the default weightings to simplify the MFAT model. We also recommend that the method of combining components be re-assessed to introduce some consistency and decrease the likelihood of unintended interactions as a result of the wide range of approaches currently being used. These recommendations should be further validated using additional data, preferably from a wider range of zones and in higher flow years than have been assessed here.

\section{ACKNOWLEDGEMENTS}

We thank the Murray-Darling Basin Authority for providing SRA fish data for use in this study and Sue Cuddy for running the baseline scenario in MFAT.

\section{REFERENCES}

Ahmadi-Nedushan, B., St-Hilaire, A., Bérubé, M., Robichaud, É., Thiémonge, N. and Bobée, B. (2006). A review of statistical methods for the evaluation of aquatic habitat suitability for instream flow assessment. River Research and Applications, 22, 503-523.

Ayllón, D., Almodóvar, A., Nicola, G.G. and Elvira, B. (2011). The influence of variable habitat suitability criteria on PHABSIM habitat index results. River Research and Applications, DOI:10.1002/rra.1496.

Bice, C. (2010) Literature Review on the Ecology of Fishes of the Lower Murray, Lower Lakes and Coorong. Report to the South Australian Department for Environment \& Heritage. South Australian Research \& Development Institute (Aquatic Sciences), Adelaide. SARDI Publication Number F2210/000031-1

Bovee, K.D. (1982). A guide to stream habitat analysis using the Instream Flow Incremental Methodology. Fish and Wildlife Service FWS/OBS-82/26. 248 pp.

Bovee, K.D. (1986). Development and evaluation of habitat suitability criteria for use in the instream flow incremental methodology. In: Instream Flow Information Paper 21. United States Fish and Wildlife Service, Cooperative Instream Flow Group. 231 pp.

Cooke, R.M. (1991). Experts in uncertainty: opinion and subjective probability in science. New York: Oxford University Press.

CSIRO (2008). Water availability in the Murray-Darling Basin. A report to the Australian Government from the CSIRO Murray-Darling Basin Sustainable Yields Project. Australia: CSIRO. 67 pp.

Davies, P.E., Harris, J.H., Hillman, T.J. and Walker, K.F. (2010). The Sustainable Rivers Audit: assessing river ecosystem health in the Murray-Darling Basin, Australia. Marine and Freshwater Research, 61, 764-777.

Jowett, I.G., Richardson, J. and Bonnett, M.L. (2005) Relationship between flow regime and fish abundances in a gravel-bed river, New Zealand. Journal of Fish Biology, 66, 1419-1436.

Korman, J., Perrin, C.J. and Lekstrum, T. (1994) A Guide for the Selection of Standrd Methods for Quantifying Sportfish Habitat Capability and Suitability in Streams and Lakes of British Columbia, Vancouver, B.C. Limnotek Research and Development Inc.

Lamouroux, N., Capra, H. and Pouilly, M. (1998). Predicted habitat suitability for lotic fish: linking statistical hydraulic models with multivariate habitat use models. Regulated Rivers: Research Management, 14, 1-11.

Marsh, N., Grigg, N. and Arene. S. (2007). A framework for capturing and applying models of biological response to natural resource management. In: MODSIM 2007, Christchurch, New Zealand.

Merritt, W.S., Powell, S.M., Pollino, C.A. and Jakeman, A.J. (2010). IBIS: a decision support tool for managers of environmental flows into wetlands. In: Ecosystem Response Modelling in the Murray-Darling Basin.--Saintilan N, Overton IC, eds. CSIRO Publishing.

Miao, S.L., Zou, C.B. and Breshears, D.D. (2009). Vegetation Responses to Extreme Hydrological Events: Sequence Matters. The Americal Naturalist, 173, 113-118.

Shuler, S.W. and Nehring, R.B. (1993). Using the physical habitat simulation model to evaluate a stream habitat enhancement project. Rivers, 4, 175-193.

SRP (2003). Ecological Assessment od Environmental Flow Reference Points for the River Murray System. Interim Report prepard by the Scientific Reference Panel for the Murray-Darling basin Commisson, Living Murray Initiative, $162 \mathrm{pp}$.

Young, W.J., Lam, D.C.L., Ressel, V. and Wong, I.W. (2000) Development of an environmental flows decision support system. Environmental Modelling and Software, 15, 257-265.

Young, W.J., Scott, A.C., Cuddy, S.M. and Rennie, B.A. (2003). Murray Flow Assessment Tool: A Technical Description (based on version 1.4). Canberra: Client Report, CSIRO Land and Water. 73. 